\title{
RESEARCH
}

\section{Obstructive sleep apnea in 2 women with familial partial lipodystrophy due to a heterozygous LMNA R482Q mutation}

\author{
Robert A. Hegele MD, Salam A. Al-Attar BSc, Brian K. Rutt PhD
}

A lthough obstructive sleep apnea has many attributes of a complex genetic trait, ${ }^{1}$ few genetic mutations have been identified in patients with the condition. Obesity has been shown to be associated with obstructive sleep apnea in the general population, ${ }^{2}$ but few rare genetic syndromes affecting total body adipose content or distribution have been evaluated for possible association with obstructive sleep apnea. Familial partial lipodystrophy subtype 2 most often results from mutations in LMNA, the gene encoding nuclear lamin A/C (MIM I5I660). Patients with familial partial lipodystrophy subtype 2 are healthy at birth, but around puberty they lose fat stores selectively in the extremities and gluteal region, while visceral, facial and neck fat stores are preserved and may enlarge with caloric excess. ${ }^{3}$ Familial partial lipodystrophy subtype 2 has been called "fat neck" syndrome in the past. ${ }^{3}$ Using polysomnography, we have documented the presence of obstructive sleep apnea in 2 women with familial partial lipodystrophy subtype 2 who each presented with daytime somnolence.

Patient I was a 49-year-old woman of normal weight in whom familial partial lipodystrophy subtype 2 had been diagnosed at age 30 years. She was $16 \mathrm{I} \mathrm{cm}$ tall, her weight was $72.5 \mathrm{~kg}$, and she had a body mass index of $27.9 \mathrm{~kg} / \mathrm{m}^{2}$, a waist circumference of $88 \mathrm{~cm}$ and blood pressure of I $45 / 88 \mathrm{~mm} \mathrm{Hg}$. Patient 2 was a 46-year-old woman of normal weight in whom the lipodystrophy had been diagnosed I year earlier. Her height was $155 \mathrm{~cm}$, weight $59.9 \mathrm{~kg}$, body mass index $25.9 \mathrm{~kg} / \mathrm{m}^{2}$, waist circumference $86 \mathrm{~cm}$ and blood pressure ir $6 / 60 \mathrm{~mm} \mathrm{Hg}$. Both patients had reasonably well-controlled diabetes and dyslipidemia. Both were heterozygous for the LMNA R $482 \mathrm{Q}$ mutation. ${ }^{4}$ The 2 women reported long-standing daytime somnolence and loud snoring at night but no witnessed episodes of obstructive sleep apnea. Neither had a history of allergies, cardiovascular disease or respiratory problems. Patient I had $6 \mathrm{fe}-$ male first- and second-degree relatives aged 40-60 years who had the LMNA R482Q mutation; patient 2 had 2 such relatives. None of these relatives had received a diagnosis of obstructive sleep apnea or had symptoms suggestive of it. Both patients had the classic familial partial lipodystrophy fat distribution, with no subcutaneous fat in the limbs and gluteal region, and increased facial, neck and central fat stores (Figure I). Neither patient had acanthosis nigricans, hirsutism or hepatomegaly, and both had normal dentition, tongue size, pharynx and palate, including uvula and tonsils. In each case, examination of the eyes and of the respiratory, cardiovascular and neurologic systems yielded normal findings, as did resting and exercise electrocardiograms. Neither patient had thyromegaly or other neck masses felt clinically, and each had normal thyroid function, as assessed by laboratory testing.

Overnight polysomnography was performed according to standard protocols (www.sleeplaboratories.com/physician-5 .htm\#5-I). Patient I's total sleep time was 332 minutes, with 32 apneic and hypopneic episodes. Patient 2's total sleep time was 39 I minutes, with 36 apneic and hypopneic episodes. The apnea-hypopnea index was 5.8 for patient I and 5.5 for patient 2, values consistent with mild obstructive sleep apnea. All apneic and hypopneic events were considered to be obstructive rather than central events.

We used a semi-automated algorithm ${ }^{5}$ to quantify the subcutaneous fat in a compartment defined as the region of the neck anterior to the rectilinear tangential line at the dorsalmost point of the paratracheal region. The proportion of the area of the anterior compartment of the neck that was adipose tissue was $56.8 \%, 68.7 \%$ and $44.1 \%$ for patient I, patient 2 and an age-and sex-matched healthy female control subject who had no lipodystrophy. Semi-automated quantification of subcutaneous fat ${ }^{5}$ from $T_{\mathrm{r}}$-weighted magnetic resonance images at vertebral level $\mathrm{C}_{5}$ showed that both patients had more than I. 6 times the adipose tissue content in the anterior compartment of the neck surrounding the trachea as compared with the control subject (Figure I).

Sleep-disordered breathing in patients I and 2 was subsequently controlled with nightly continuous positive airway pressure of IO and $5 \mathrm{~cm} \mathrm{H}_{2} \mathrm{O}$, respectively, administered by nasal mask. Both patients reported marked improvement in their symptoms.

Charcot-Marie-Tooth disease, another monogenic disorder, is also associated with obstructive sleep apnea, ${ }^{6}$ but

From the Vascular Biology Research Group (Hegele, Al-Attar) and Imaging Research Laboratories (Rutt), Robarts Research Institute, London, Ont. 


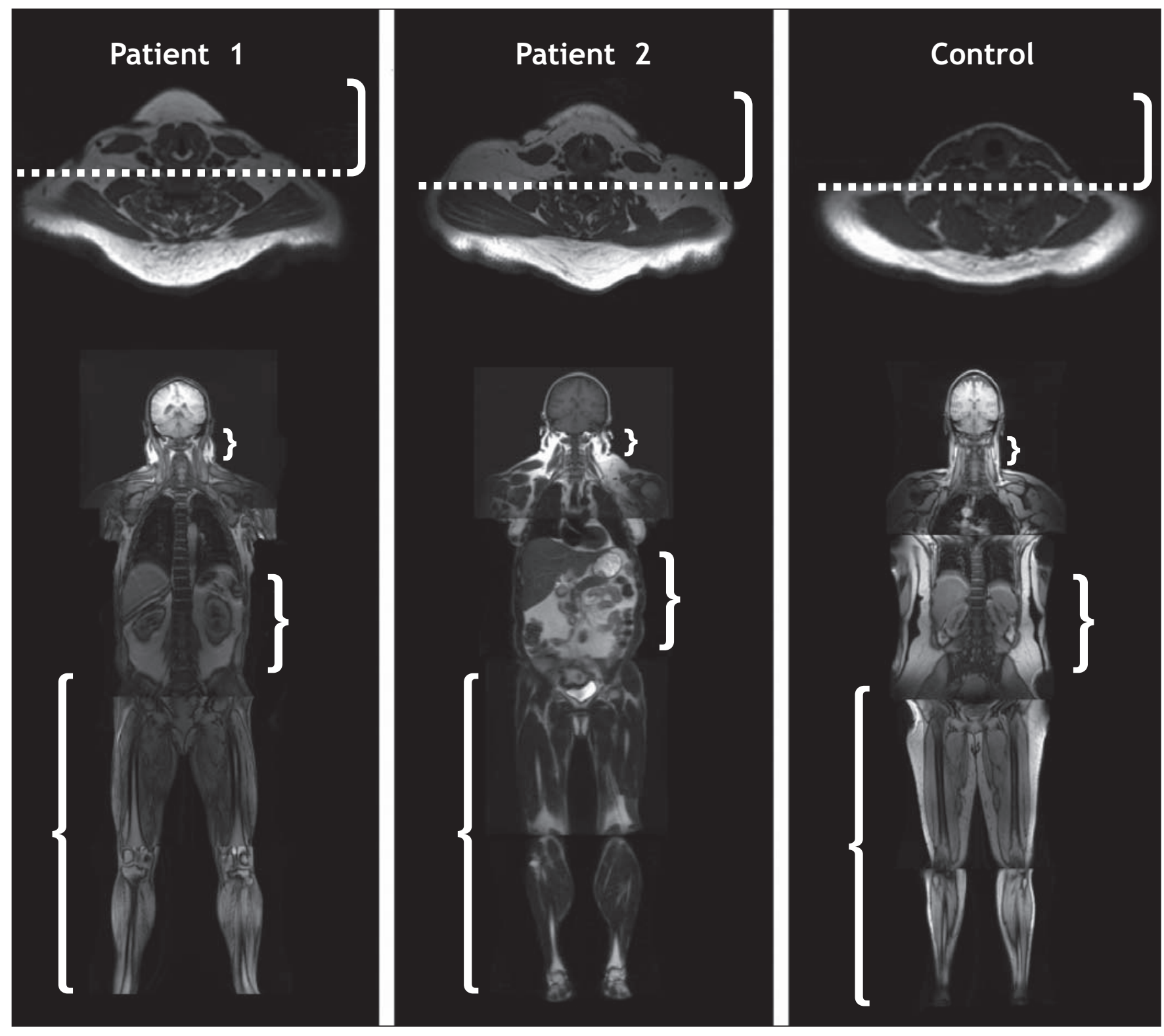

Figure 1: Top panels: $T_{1}$-weighted cross-sectional magnetic resonance images at vertebral level $C_{5}$ for patients 1 and 2 and an age- and sex-matched female control subject who did not have lipodystrophy. Subcutaneous neck fat was quantified in a compartment defined by the bracket and anterior to the rectilinear tangential line (dotted line) at the dorsal-most point of the paratracheal region. Bottom panels: corresponding $T_{1}$-weighted magnetic resonance images of the whole body. Patients 1 and 2 were not obese; in fact in the lower body region (within the large bracket) they had a deficiency of subcutaneous fat compared with the control subject. However, patients 1 and 2 had excess intra-abdominal fat (region within the medium bracket) and neck fat (region within the small bracket) compared with the control subject.

the sleep apnea in lipodystrophy is not associated with neuropathy or muscle weakness. Furthermore, obstructive sleep apnea has been reported in patients with acquired lipodystrophy syndromes, specifically partial lipodystrophy associated with HIV infection treated with highly active antiretroviral drugs. ${ }^{7}$ No clear mechanism links familial partial lipodystrophy subtype 2 with obstructive sleep apnea, although both of the patients we have described were of normal weight but had markedly increased neck fat content, specifically in the region surrounding the trachea. This sug- gests that the association might be related to the repartitioning of adipose tissue that is characteristic of patients with this type of lipodystrophy.

Although the obstructive sleep apnea in these 2 cases may have been coincidental, it is important to note that neither of the patients was obese and each had clinically ascertained lipodystrophy. Anecdotally, we have heard of additional cases of sleep apnea in extended kindreds. A more systematic assessment of the presence of obstructive sleep apnea in people with familial partial lipodystrophy or other lipodystrophies 
would increase confidence in the possible mechanistic association of these conditions.

This article has been peer reviewed.

Competing interests: None declared.

Contributors: All of the authors contributed to the conception and design of the study or the acquisition or analysis and interpretation of data. All drafted or revised the article critically for important intellectual content and approved the final version to be published.

Acknowledgements: Robert Hegele is supported by the Jacob J. Wolfe Distinguished Medical Research Chair, the Edith Schulich Vinet Canada Research Chair (Tier I) in Human Genetics, a Career Investigator award from the Heart and Stroke Foundation of Ontario, and operating grants from the Canadian Institutes for Health Research, the Heart and Stroke Foundation of Ontario, the Ontario Research Fund and by Genome Canada through the Ontario Genomics Institute.

\section{REFERENCES}

I. Redline S, Tishler PV. The genetics of sleep apnea. Sleep Med Rev 2000;4:583-602.

2. Wolk R, Somers VK. Obesity-related cardiovascular disease: implications of obstructive sleep apnea. Diabetes Obes Metab 2006;8:250-60.

3. Hegele RA. Monogenic forms of insulin resistance: apertures that expose the common metabolic syndrome. Trends Endocrinol Metab 2003;I4:37I-7.

4. Cao H, Hegele RA. Nuclear lamin A/C R482Q mutation in Canadian kindreds with Dunnigan-type familial partial lipodystrophy. Hum Mol Genet 2000;9:I09-I2.

5. Al-Attar SA, Pollex RL, Robinson JF, et al. Semi-automated segmentation and quantification of adipose tissue in calf and thigh by MRI: a preliminary study in patients with monogenic metabolic syndrome. BMC Med Imaging 2006;6:II

6. Dematteis M, Pepin JL, Jeanmart M, et al. Charcot-Marie-Tooth disease and sleep apnoea syndrome: a family study. Lancet 200I;357:267-72.

7. Lo Re V III, Schutte-Rodin S, Kostman JR. Obstructive sleep apnoea among HIV patients. Int J STD AIDS 2006;17:6I4-20.

Correspondence to: Dr. Robert A. Hegele, Blackburn

Cardiovascular Genetics Laboratory, Robarts Research Institute, London ON N6A 5K8; fax 519 663-3037; hegele@robarts.ca 